\title{
COMBINED $L_{2}-L_{1}$-NORM REGULARIZATION IN FLUORESCENCE DIFFUSE OPTICAL TOMOGRAPHY
}

\author{
An Jin, Birsen Yazıcl \\ Rensselaer Polytechnic Institute \\ 110 8th St., Troy, NY 12180
}

\begin{abstract}
The inverse problem of fluorescence diffusse optical tomography (FDOT) is often highly ill-posed, which needs regularization techniques. In this paper, we propose a combined $l_{1}$ $l_{2}$-norm regularization method to address the ill-posed FDOT inverse problem. Compared with the traditional Tikhonov regularization, the proposed method is able to effectively remove the noise in the reconstructed image without much over-smoothness. The performance of the proposed method is demonstrated in 3D numerical simulation.
\end{abstract}

Index Terms - optical imaging, reconstruction

\section{INTRODUCTION}

The inverse problem of fluorescence diffusse optical tomography (FDOT) requires reconstruction of $2 \mathrm{D}$ or $3 \mathrm{D}$ fluorophore map inside the imaging domain using boundary measurements obtained at the emission and excitation wavelengths [1]. However, the number of measurements available is usually insufficient. Furthermore, the solution is not stable, i.e., a small perturbation in the measurement data can result in large deviations in the solution [1]. Thus, regularization techniques are usually necessary.

In the FDOT, the fluorophore yield, which is proportional to the fluorophore concentration, often has large value in a small foreground region, and is close to zero in the background [2]. The most widely used Tikhonov regularization with a quadratic regularization function imposes heavy penalty on large argument values, which often leads to oversmoothing in the foreground region [3]. A regularization function that increases less rapidly for large argument values than quadratic function works better to preserve the foreground region $[2,4]$. However, such penalty function might be insufficient in smoothing out large spikes of noise, which is possible to exit in the reconstructed image due to the illposedness of the forward matrix and the measurement noise. In this work, we propose a new regularization function that combines both the quadratic regularization and the $l_{1}$-norm regularization, which we refer to as the combined $l_{2}-l_{1}$-norm regularization in the following discussion. The proposed method is capable of preserving large argument values in the foreground region while effectively removing noise in the reconstructed image. We demonstrate the performance of the proposed method by $3 \mathrm{D}$ numerical simulation.

\section{FDOT IMAGING PROBLEM}

The FDOT inverse problem involves reconstructing unknown fluorophore yield, which is proportional to the fluorophore concentration, from the boundary measurements. Given $N_{s}$ sources and $N_{d}$ detectors, the measurement $\Gamma_{i, j}$ of the $j$ th detector due to $i$ th source is given as follows [1],

$$
\Gamma_{i, j}=\int_{\Omega} g_{j}^{*}(\boldsymbol{r}) \phi_{i}(\boldsymbol{r}) \mu(\boldsymbol{r}) d \boldsymbol{r}
$$

where $\Omega$ is the imaging domain, $\phi_{i}(\boldsymbol{r})$ is the excitation light field due to the $i$ th source, $g_{j}^{*}(\boldsymbol{r})$ is the Greens function of the $j$ th detector, and $\mu(\boldsymbol{r})$ is the fluorophore yield. We discretize the domain into $N$ voxels, and obtain the discretized form,

$$
\Gamma=A \mu,
$$

where $\boldsymbol{y} \in \mathbb{R}^{M}\left(M=N_{s} \times N_{d}\right)$ is the measurement vector, $\boldsymbol{A} \in \mathbb{R}^{M \times N}$ is the vector-valued forward operator, and $\boldsymbol{\mu} \in$ $\mathbb{R}^{N}$ is the discretized fluorophore yield.

Regularization techniques are often applied to solve for $\boldsymbol{\mu}$, which trades off the quadratic error of the measurement with a regularization function $R(\boldsymbol{\mu})$,

$$
\hat{\boldsymbol{\mu}}=\arg \min \boldsymbol{\mu}\|\boldsymbol{y}-\boldsymbol{A} \boldsymbol{\mu}\|^{2}+\lambda R(\boldsymbol{\mu}),
$$

where $\lambda$ is the regularization parameter. If $R(\boldsymbol{\mu})$ is continuous and differentiable, (3) can be easily solved by gradient based method, such as the nonlinear conjugate gradient method [5].

\section{THE COMBINED $L_{2}-L_{1}$-NORM REGULARIZATION}

In this section, we propose a combined $l_{2}-l_{1}$-norm regularization. Assume that the amplitude of $\boldsymbol{\mu}$ in the foreground region are approximately in a known range $[\alpha, \beta]$, which can be determined from empirical values. The combined $l_{2}-l_{1}$-norm regularization imposes small penalty when $\alpha \leq \mu_{i} \leq \beta$, and 
large penalty when $\mu_{i} \notin[\alpha, \beta]$. The regularization function $R(\boldsymbol{\mu})$ is the summation of the cost functions of each voxel,

$$
R(\boldsymbol{\mu})=\sum_{i=1}^{N} r\left(\mu_{k}\right),
$$

where $r\left(\mu_{k}\right)$ is the cost function of the $\mu_{k} . r\left(\mu_{k}\right)$ has the following properties: (i) $r\left(\mu_{k}\right)$ is continuous and differentiable, $\forall \mu_{k} \in \mathbb{R}$, (ii) $r\left(\mu_{k}\right)$ has large quadratic penalty when $\mu_{k} \notin$ $[\alpha, \beta]$, (iii) $r\left(\mu_{k}\right)$ has $l_{1}$-norm penalty when $\mu_{k} \in[\alpha, \beta]$. Based on the properties listed above, $r\left(\mu_{k}\right)$ is given by

$$
r\left(\mu_{k}\right)= \begin{cases}\frac{a \mu_{k}^{2}}{2 \epsilon}, & \left|\mu_{k}\right| \leq \epsilon ; \\ a\left|\mu_{k}\right|-\frac{a \epsilon}{2}, & \epsilon \leq\left|\mu_{k}\right| \leq \epsilon^{\prime} ; \\ c_{2} \mu_{k}^{2}+c_{1}\left|\mu_{k}\right|+c_{0}, & \left|\mu_{k}\right|>\epsilon^{\prime},\end{cases}
$$

where $\epsilon$ and $\epsilon^{\prime}$ are two constants satisfying $0<\epsilon<\alpha<\epsilon^{\prime}<$ $\beta$. $a, c_{0}, c_{1}$ and $c_{2}$ are constant parameters chosen such that $R(\boldsymbol{\mu})$ is continuous and differentiable,

$$
\begin{array}{r}
a=\left(2 \lambda \alpha^{2}\right) /(2 \alpha-\epsilon), \\
c_{2}=\left(\lambda \beta^{2}-a \beta+\frac{a \epsilon}{2}\right) /\left(\beta-\epsilon^{\prime}\right)^{2}, \\
c_{1}=a-2 \epsilon^{\prime} c_{2}, \\
c_{0}=c_{2} \epsilon^{\prime 2}-\frac{a \epsilon}{2} .
\end{array}
$$

\section{NUMERICAL SIMULATION RESULTS}

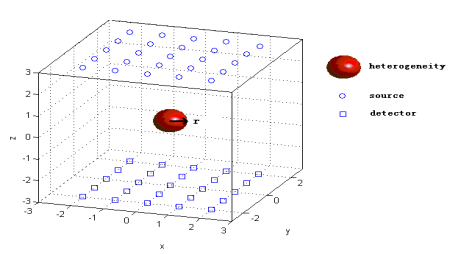

Fig. 1. Phantom configuration.

In this section, we present $3 \mathrm{D}$ numerical simulation results. The simulated imaging domain is a $6 \times 6 \times 6 \mathrm{~cm}^{3} \mathrm{cu}-$ bic region, with sources and detectors uniformly distributed, as shown in Fig.1. The measurements are corrupted by i.i.d Gaussian noise with $30 d B$ signal to noise ration (SNR). The fluorophore is concentrated in the center of radius $0.5 \mathrm{~cm}$.

Fig.2(a) shows the cross section of the original phantom. Reconstruction results of the Tikhonov regularization is shown in Fig.2(b) with severer over-smoothing. Fig.2(c) shows the reconstruction result of the proposed combined $l_{1}-l_{2}$-norm regularization. Fig.2(c) has much less oversmoothness and clear foreground region.

The mean square error (MSE) between the reconstructed fluorophore map and the original phantom is shown in Fig.3. The combined $l_{2}-l_{1}$-norm regularization has a much lower

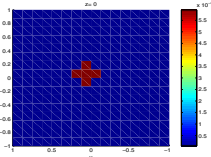

(a) Phantom

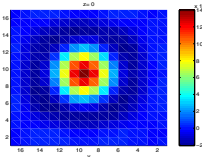

(b) Tikhonov

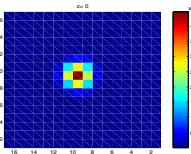

(c) Combined $l_{2}-l_{1}-$ norm
Fig. 2. Cross section of the original phantom and the reconstruction results.

MSE compared to the Tikhonov regularization at different SNR levels. The MSE analysis matches visual results.

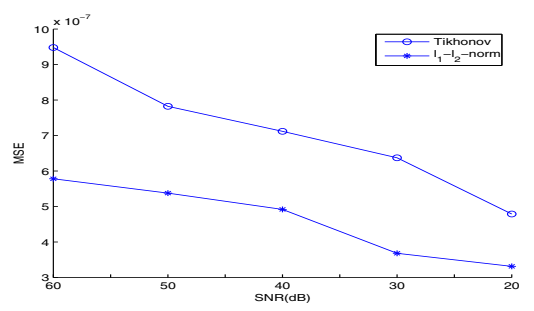

Fig. 3. MSE-SNR plot of the reconstruction results.

\section{CONCLUSION}

In this work, we propose a combine $l_{1}-l_{2}$-norm regularization technique to address the ill-posed FDOT inverse problem. Numerical results show that the proposed method is able to preserve the fluorophore region, and effectively remove large spike of noise without much over-smoothing.

\section{REFERENCES}

[1] A. Milstein, D. Boas, R. Millane, C. Bouman, and $\mathrm{K}$. Webb, "Fluorescence optical diffusion tomography using multiple-frequency data," Journal of the Optical Society of America A, vol. 21(6), pp. 1035-1049, 2004.

[2] J. Huang P. Mohajerani, A. Eftekhar and A. Adibi, "Optimal sparse solution for fluorescent diffuse optical tomography: theory and phantom experimental results," $A p$ plied Optics, vol. 46(10), pp. 1679-1685, 2007.

[3] B. Pogue, T.McBride, J. Prewitt, U. Osterberg, and K. Paulsen, "Spatially variant regularization improves diffuse optical tomography," Appl. Opt., vol. 38, pp. 2950-2961, 1999.

[4] M. Suzen, A. Giannoula, and T. Durduran, "Compressed sensing in diffuse optical tomography," Optics Express, vol. 18(23), pp. 23676-23690, 2010.

[5] J.Shewchuk, "An introduction to the conjugate gradient method without the agonizing pain," 1994. 